American Journal of Infectious Diseases 2 (4): 204-209, 2006

ISSN 1553-6203

(C) 2006 Science Publications

\title{
Applications of Real-time Reverse Transcription Polymerase Chain Reaction in Clinical Virology Laboratories for the Diagnosis of Human Diseases
}

\author{
${ }^{1}$ R. Manojkumar and ${ }^{2}$ V. Mrudula \\ ${ }^{1}$ Department of Microbiology and Immunology, \\ ${ }^{2}$ Department of Pathology, New York Medical College, Valhalla, NY-10595, New York, USA
}

\begin{abstract}
Early diagnosis that prevents further expansion of the causative agent and thereby aids control and eradication of infectious agents is critical for countries trying to obtain a particular disease free status. In these diagnostics speed is paramount, including the assay's applicability, sensitivity, specificity, cost effectiveness and patentability. Real-time Reverse Transcription PCR (rRT-PCR) has revolutionized the field of molecular biology and is being utilized increasingly in novel clinical diagnostic assays. This technique has been applied for rapid detection of point mutation, drug resistant variant and genotyping in several viruses. The combination of excellent sensitivity, specificity, low contamination risk, and speed has made this technique an appealing alternative to cell culture or immunoassay-based testing methods for diagnosing infectious diseases. rRT-PCR assays are most established for the detection of viral load and therapy monitoring. In this review, the usefulness or applications of rRT-PCR assays in the diagnosis of some of the important human viral infections have been summarized.
\end{abstract}

Keywords: Early diagnosis, polymerase chain, virology,

\section{INTRODUCTION}

Real-time Reverse Transcription Polymerase Chain Reaction (rRT-PCR) has originated as a powerful tool for quantitative nucleic acids analysis ${ }^{[1,2]}$. rRTPCR techniques are increasingly used to quantify RNA viruses for diagnosis ${ }^{[3]}$, the standard for the detection and quantification of RNA targets ${ }^{[4]}$ and is firmly established as a mainstream research technology ${ }^{[5]}$. This technique is a refinement of the original PCR developed by Kary Mullis and co-workers in the mid 1980 's ${ }^{[5,6]}$. It's potential for high-throughput; together with regular introduction of enhanced or novel chemistries, more reliable instrumentation and improved protocols ${ }^{[7,8]}$ made it the choice of diagnostics. The principle of rRT-PCR assays is as follows, the RNA will be reverse transcriped into cDNA, and it requires a suitable detection system for reporting the presence of PCR products, to monitor the amplification in real-time and the software programs for quantitative analysis ${ }^{[9]}$. The amplification is detected by detection chemistries which can be either probe (specific) or non-probe (non-specific) based, and are discussed elaborately ${ }^{[10]}$. The gene-specific probes labeled for different genomic regions are better utilized in diagnosis $[11,12,13]$. In rRT-PCR the amount of product formed is monitored during the course of the reaction by monitoring the fluorescence of dyes or probes introduced into this reaction is proportional to the amount of product formed, and the number of amplification cycles required to obtain a particular amount of DNA molecules ${ }^{[2]}$.

Some of the advantages of using rRT-PCR are as follows, 1] traditional PCR is measured at end-point, while rRT-PCR collects data in the exponential growth phase, 2] an increase in reporter fluorescent signal is directly proportional to the number of amplicons generated, 3] the cleaved probe provides a permanent record amplification of an amplicon, 4] increased dynamic range of detection, 5] requirement of 1000fold less RNA than conventional assays, 6] no-post PCR processing due to closed system, 7] detection ranges down to a 2-fold change, 8] small amplicon size results in increased amplification efficiency, and 9] Less time-consuming. rRT-PCR can be applied to traditional PCR applications as well as new applications that would have been less effective with traditional

Corresponding Author: Dr. R. Manojkumar, Department of Microbiology and Immunology, Basic Sciences, Building, New York Medical College, Valhalla, NY-10595, USA 
PCR. With the ability to collect data in the exponential growth phase, the power of rRT-PCR has been expanded into applications such as, a] Quantitation of gene expression ${ }^{[14]}$ including NK cell KIR gene expression [15], b] Drug therapy efficacy/drug monitoring [16], c] Viral quantitation [17, 18], and d] Pathogen detection ${ }^{[19,20]}$. To identify new foci of the disease, it is necessary to harness diagnostic methods, both rapid and sensitive, for differentiating reliably field viruses from vaccine strains. rRT-PCR when compared to ELISA, RT-PCR and Virus Isolation in cell culture, have greater versatility, sensitivity and specificity. By using rRT-PCR, the disadvantages of conventional PCR methods concerning the risk of contamination and the lack of specificity controls are minimized. The technique was and is being used in the diagnosis of a wide variety of diseases caused by RNA viruses in humans and some of them are being summarized here.

Applications of rRT-PCR as diagnostic: RNA viruses constitute the most abundant group of pathogens in man, and are classified according to their RNA genome. The low efficiency of proofreading and error prone post-replicative repair activities of viral RNA polymerases results in high mutation rates, causing potential problems for an assay that relies on exquisite sequence specificity ${ }^{[10]}$. The ability of rRT-PCR to generate accurate quantitative data has a huge impact on the study of viral agents of infectious disease ${ }^{[21]}$.

Positive-stranded RNA viruses: The genome of positive-stranded RNA viruses consists of a single stranded Sense RNA and carries its own RNAdependent RNA polymerase enzyme. The RNA is initially translated by the host enzymes and then catalyses the synthesis of large numbers of anti-sense replicative intermediates which in turn serve as templates for the synthesis of a large number of mRNA molecules. This group includes some of the important pathogens like the entero-, rhino-, hepato-, hepaci-, corona-, dengu viruses etc.,.

The incidence of enterovirus infections was studied with the help of rRT-PCR using blood, feces, and cerebrospinal fluid (csf) samples collected from infants and the assay finds efficient in appropriate diagnosis of enterovirus infection ${ }^{[22,23]}$. The detection of the enterovirus genome in csf has proved to be more sensitive than traditional cell culture. Hence, a one-step rRT-PCR assay developed with LightCycler (LC) technology allowed the detection of enterovirus in csf in $3 \mathrm{hrs}$ as opposed to $36 \mathrm{hrs}$ of regular RT-PCR assay [24]. In another study, a single-tube, rRTPCR/hybridization assay using TaqMan with LC technology was optimized for enterovirus screening that was carried out in $2 \mathrm{hrs}{ }^{[25]}$. Enterovirus 71 (EV71) is one of the main causative agents of hand, foot and mouth disease in young children ${ }^{[26]}$ and a rapid rRTPCR hybridization probe assay targeting the VP1 region of EV71 showed that the assay could be applied for specific detection of EV71 directly from clinical samples ${ }^{[26]}$.

The rhinoviruses are the major causative agent of common cold affecting the human respiratory system. The initial detection of rhinovirus was carried out by VI in cell culture which was further improved by the use of RT-PCR assays. Further, the rRT-PCR assay increased the detection sensitivity to 10 -fold that of the conventional RT-PCR assays ${ }^{[27]}$. Simultaneous detection and quantification of both the enteroviruses and rhinoviruses was carried out efficiently by rRTPCR assay ${ }^{[28]}$. Diagnosis of Hepatitis $A$ and $C$ viruses (HAV, HCV) employs rRT-PCR assays for rapidity and accuracy. In a one-step rRT-PCR Taqman assay to quantify the $5^{\prime}$ non-coding region of HAV ${ }^{[3]}$, for routine genotyping of $\mathrm{HCV}{ }^{[29,30]}$ and a multiplex quantitative rRT-PCR assay for simultaneous detection, identification and quantification of hepatitis $\mathrm{B}$ virus (HBV-DNA virus), $\mathrm{HCV}$ and human immunodeficiency virus type 1 (HIV-1) in plasma or serum samples using flourogenic probes showed increased sensitivity and accuracy ${ }^{[31,32]}$, which proves its potentiality to be used for large-scale nucleic acid testing of blood donations ${ }^{[30]}$.

Severe Acute Respiratory Syndrome (SARS) is a highly infectious disease with significant morbidity and case fatality. The outbreak of SARS was caused by a newly identified coronavirus SARS-CoV in $2003^{\text {[33] }}$. Although conventional RT-PCR is useful in the identification of the SARS virus, the assays were not sufficiently sensitive in diagnosing the conditions in the first few days after disease onset, which is the period during which laboratory diagnosis is most relevant for patient care ${ }^{[10]}$. Hence, rRT-PCR-based diagnostic assays were rapidly developed ${ }^{[34,35]}$ with improved specificity of $100 \%$ in samples collected from 1-3 days of disease onset [36, 37]. Recently, quantitative 
measurement of blood SARS CoV RNA using rRTPCR showed a detection rate of $80 \%$ as early as day 1 in hospital patients ${ }^{[33]}$. The absolute quantitation of human coronaviruses OC43 and 229E [38], simultaneously detection of nucleo-capsid, membrane, and spike genes of SARS-CoV with same PCR conditions ${ }^{[39]}$ and the improved sensitivity of the recently established real-time loop-mediated amplification systems. Poon et al., ${ }^{[40]}$ concluded that the assays might be a possible solution for on-site diagnosis for SARS Co V detection.

Dengue fever and dengue hemorrhagic fever (DHF)/dengue shock syndrome are important human arboviral diseases caused by infection with one of four closely related but serologically distinct viruses, designated as DEN-1, 2, 3, and 4 viruses ${ }^{[41]}$. Since there is no protective vaccine or specific treatments available for dengue fever/DHF, accurate diagnosis is critical for the early initiation of specific preventive health measures to curtail epidemic spread and reduce economic losses ${ }^{[42]}$. Typing of dengue virus is crucial for epidemiology and study of pathogenesis. Hence, highly sensitive and accurate diagnostic tools are essential. A serotype-specific, fourplex rRT-PCR assay is being used as a method for differential diagnosis of a specific dengue serotype in viremic stage ${ }^{[41]}$. Another rRT-PCR assay targeting the 3'-untranslated region of this virus using SYBR Green I as the detecting system was used to identify all four types ${ }^{[43]}$.

Negative-stranded RNA viruses: The genome of negative-stranded RNA viruses consists of singlestranded anti-sense RNA. These are transcribed into sense-strand mRNA by RNA-dependent RNA polymerases carried by the viruses themselves. This group includes the measles, mumps, influenza, respiratory syncytial virus and parainfluenza virus types $1-4{ }^{[10]}$ which cause respiratory infections of the upper and lower respiratory tract in infants and young children and some other viruses producing viral hemorrhagic fevers (VHFs). VHFs are acute infections with high case fatality rates. The important agents causing VHFs are Ebola and Marburg viruses, Lassa virus, Crimean-Congo Hemorrhagic Fever virus, Rift Valley fever virus and the positive strand RNA viruses like Dengue virus and Yellow Fever virus. Since VHFs are clinically difficult to diagnose and distinguish, a rapid and reliable laboratory diagnostics are needed for suspected cases. Hence, a one-step, rRT-PCR assay using the superscript reverse transcriptase-Platinum Taq polymerase enzyme was established for the detection and quantification of Ebola viral RNA and the assay proved very effective in detecting Ebolavirus in patient's serum, plasma, and whole blood ${ }^{[44]}$. The assay was also used to determine the viral load in multiple samples from patients with fatal and nonfatal cases. Furthermore, rRT-PCR was applied for testing the potency of the antivirals against the highly pathogenic Ebola virus ${ }^{[45]}$. Another group of viruses causing hemorrhagic fever are Hantaan viruses and their various types (Dobrava, Hantaan, Puumala, and Seoul viruses). rRT-PCR assay was developed which is useful for diagnosing and differentiating these four Hantaviruses ${ }^{\text {[46] }}$. A fast method using quantitative rRT-PCR assay has been developed to measure the potency of measles in trivalent measles, mumps and rubella (MMR) vaccines and validated to estimate the actual viral antigen load in the vaccine ${ }^{[47]}$.

Double-stranded RNA viruses: This group of viruses has a segmented double stranded (ds) RNA genome, together with a RNA-dependent RNA polymerase that transcribes each of the ds RNA molecules into an mRNA ${ }^{[10]}$. This group includes the most important human rotaviruses which cause gastro-enteric problems in infants and young children. The enhanced sensitivity and a lower risk for cross-contamination in rRT-PCR makes it a useful tool for the detection of rotavirus in various situations including sporadic gastroenteritis, outbreaks, and environmental investigations ${ }^{[48]}$. A competitive rRT-PCR ${ }^{[49]}$ and a semi-quantitative rRTPCR assays ${ }^{[50]}$ using SYBR Green I dye was developed 
to detect and to quantitate the RNA of group A rotaviruses, and to estimate the rotavirus load in diarrhoeal samples, respectively. These assays were very efficient as it could detect even small amounts of viral RNA of group A rotaviruses in clinical samples obtained from humans.

Retroviruses: Nucleic acid extraction by magnetic micro beads followed by rRT-PCR founds to be a reliable, sensitive, rapid and simple procedure to quantify HIV-1 RNA in breast milk samples [51]. Monitoring the plasma RNA viral load in HIV-1 group $\mathrm{O}$ infection was found to be difficult before the same has been carried out by rRT-PCR assay ${ }^{[52]}$.

\section{CONCLUSION}

The newly established methods must be standardized to maintain high quality laboratory performance. Laboratory diagnostics must be tailored to a specific laboratory environment, the objectives of clinical needs and the availability of clinical specimens. Speed and accuracy of diagnosis must be balanced against test cost and availability. Future challenges in the study of human viral diseases include the application of modern techniques, such as nucleic acid chips, protein chips and new biomarkers to avoid crossreactivity among different samples, strains or serotypes, as well as development of internationally standardized guidelines to improve quality assurance of these advanced laboratory tests.

\section{REFERENCES}

1. Gibson, U.E., C.A. Heid and P.M. Williams, 1996. A novel method for real-time quantitative RT-PCR. Genome Res., 6: 995-1001.

2. Kubista, M., J.M. Andrade and M. Bengtsson et al., 2006. The real-time polymerase chain reaction. Mol. Aspects Med., 27: 95-125.
3. Costa-Mattioli, M., S. Monpoeho and E. Nicand et al., 2002. Quantification and duration of viraemia during hepatitis A infection as determined by real-time RT-PCR. J. Viral Hepat., 9: 101-106.

4. Bustin, S.A., 2000. Absolute quantification of mRNA using real-time reverse transcription polymerase chain reaction assays. J. Mol. Endocrinol., 25: 169-193.

5. Ginzinger, D.G., 2002. Gene quantification using real-time quantitative $\mathrm{PCR}$, an emerging technology hits the mainstream. Exp. Hematol., 30: 503-512.

6. Wright, P.A. and D. Wynford-Thomas, 1990. The polymerase chain reaction, miracle or mirage? A critical review of its uses and limitations in diagnosis and research. J. Pathol., 162: 99-117.

7. Nashed, A.L., K.W. Rao and M.L. Gulley, 2003. Clinical applications of BCR-ABL molecular testing in acute leukemia. J. Mol. Diagn., 5: 6372.

8. Niesters, H.G., 2004. Molecular and diagnostic clinical virology in real-time. Clin. Microbiol. Infect., 10: 5-11.

9. Wittwer, C.T., M.G. Herrmann and A.A. Moss et al., 1997. Continuous fluorescence monitoring of rapid cycle DNA amplification. BioTechniques, 22: 130-138.

10. Bustin, S.A., and R. Mueller, 2005. Real-time reverse transcription PCR (qRT-PCR) and its potential use in clinical diagnosis. Clin. Sci., 109: 365-379.

11. Oem, J.K., S.J. Kye and K.N. Lee et al., 2005. Development of a Lightcycler-based reverse transcription polymerase chain reaction for the detection of foot-and-mouth disease virus. J. Vet. Sci., 6: 207-212.

12. Oleksiewicz, M.B., A.I. Donaldson and S. Alexandersen, 2001. Development of a novel real-time RT-PCR assay for quantitation of footand-mouth disease virus in diverse porcine tissues. J. Virol. Methods, 92: 23-35. 
13. Reid, S.M., N.P. Ferris and G.H. Hutchings et al., 2002. Detection of all seven serotypes of foot-and-mouth disease virus by real-time, fluorogenic reverse transcription polymerase chain reaction assay. J. Virol. Methods, 105: 6780.

14. Giulietti, A., L. Overbergh and D. Valckx et al., 2001. An overview of real-time quantitative PCR, applications to quantify cytokine gene expression. Methods, 25: 386-401.

15. Leung, W., R. Iyengar and B. Triplett et al., 2005. Comparison of Killer Ig-Like Receptor Genotyping and Phenotyping for Selection of Allogeneic Blood Stem Cell Donors. J. Immunol., 174: 6540-6545.

16. Leruez-Ville, M., V. Minard and F. Lacaille et al., 2004. Real-time blood plasma polymerase chain reaction for management of disseminated adenovirus infection. Clin. Infect. Dis., 38: 4552.

17. Mengelle, C., C. Pasquier and L. Rostaing et al., 2003. Quantitation of human cytomegalovirus in recipients of solid organ transplants by real-time quantitative PCR and pp65 antigenemia. J. Med. Virol., 69: 225-231.

18. Niesters, H.G., 2001. Quantitation of viral load using real-time amplification techniques. Methods, 25: 419-429.

19. Mackay, I.M., 2004. Real-time PCR in the microbiology laboratory. Clin. Microbiol. Infect., 10: 190-212.

20. Watzinger, F., M. Suda and S. Preuner et al., 2004. Real-time Quantitative PCR Assays for Detection and Monitoring of Pathogenic Human Viruses in Immunosuppressed Pediatric Patients. J. Clin. Microbiol., 42: 5189-5198.

21. Schutten, M. and H.G. Niesters, 2001. Clinical utility of viral quantification as a tool for disease monitoring. Expert Rev. Mol. Diagn., 1: 153162.

22. Verboon-Maciolek, M.A., M. Nijhuis and A.M. van Loon et al., 2003. Diagnosis of enterovirus infection in the first 2 months of life by real-time polymerase chain reaction. Clin. Infect. Dis., 37: $1-6$.
23. Verstrepen, W.A., S. Kuhn and M.M. Kockx et al., 2001. Rapid detection of enterovirus RNA in cerebrospinal fluid specimens with a novel single-tube real-time reverse transcription-PCR assay. J. Clin. Microbiol., 39: 4093-4096.

24. Archimbaud, C., A. Mirand and M. Chambon et al., 2004. Improved diagnosis on a daily basis of enterovirus meningitis using a one-step real-time RT-PCR assay. J. Med. Virol., 74: 604-611.

25. Watkins-Riedel, T., M. Woegerbauer and D. Hollemann et al., 2002. Rapid diagnosis of enterovirus infections by real-time PCR on the LightCycler using the TaqMan format. Diagn. Microbiol. Infect. Dis., 42: 99-105.

26. Tan, E.L., V.T. Chow and G. Kumarasinghe et al., 2006. Specific detection of enterovirus 71 directly from clinical specimens using real-time RT-PCR hybridization probe assay. Mol. Cell. Probes, 20: 135-140.

27. Dagher, H., H. Donninger and P. Hutchinson et al., 2004. Rhinovirus detection, comparison of real-time and conventional PCR. J. Virol. Methods, 117: 113-121.

28. Kares, S., M. Lonnrot and P. Vuorinen et al., 2004. Real-time PCR for rapid diagnosis of entero- and rhinovirus infections using LightCycler. J. Clin. Virol., 29: 99-104.

29. Lindh, M. and C. Hannoun, 2005. Genotyping of hepatitis $\mathrm{C}$ virus by Taqman real-time PCR. J. Clin. Virol., 34: 108-114.

30. Rolfe, K.J., G.J. Alexander and T.G. Wreghitt et al., 2005. A real-time Taqman method for hepatitis C virus genotyping. J. Clin. Virol., 34: 115-121.

31. Candotti, D., J. Temple and S. Owusu-Ofori et al., 2004. Multiplex real-time quantitative RTPCR assay for hepatitis B virus, hepatitis C virus, and human immunodeficiency virus type 1. J. Virol. Methods, 118: 39-47.

32. Gibellini, D., F. Gardini and F. Vitone et al., 2006. Simultaneous detection of HCV and HIV1 by SYBR Green real-time multiplex RT-PCR technique in plasma samples. Mol. Cell. Probes, (In press). 
33. Hui, D.S., P.C. Wong and C. Wang, 2003. SARS, clinical features and diagnosis. Respirology, 8: 20-24.

34. Poon, L.L., K.H. Chan and O.K. Wong et al., 2003a. Early diagnosis of SARS coronavirus infection by real-time RT-PCR. J. Clin. Virol., 28: 233-238.

35. Poon, L.L., O.K Wong and K.H. Chan et al., 2003b. Rapid diagnosis of a coronavirus associated with severe acute respiratory syndrome (SARS). Clin. Chem., 49: 953-955.

36. Hui, R.K., F. Zeng and C.M. Chan et al., 2004. Reverse transcriptase PCR diagnostic assay for the coronavirus associated with severe acute respiratory syndrome. J. Clin. Microbiol., 42: 1994-1999.

37. Poon, L.L., B.W. Wong and K.H. Chan et al., 2004. A one step quantitative RT-PCR for detection of SARS coronavirus with an internal control for PCR inhibitors. J. Clin. Virol., 30: 214-217.

38. Vijgen, L., E. Keyaerts and E. Moes et al., 2005. Development of one-step, real-time, quantitative reverse transcriptase PCR assays for absolute quantitation of human coronaviruses OC43 and 229E. J. Clin. Microbiol., 43: 5452-5456.

39. Huang, J.L., H.T. Lin and Y.M. Wang et al., 2005. Rapid and sensitive detection of multiple genes from the SARS-coronavirus using quantitative RT-PCR with dual systems. J. Med. Virol., 77: 151-158.

40. Poon, L.L., B.W. Wong and H.K. Chan et al., 2005. Evaluation of real-time reverse transcriptase PCR and real-time loop-mediated amplification assays for severe acute respiratory syndrome coronavirus detection. J. Clin. Microbiol., 43: 3457-3459.

41. Johnson, B.W., B.J. Russell and R.S. Lanciotti, 2005. Serotype-specific detection of dengue viruses in a fourplex real-time reverse transcriptase PCR assay. J. Clin. Microbiol., 43: 4977-4983.

42. Kao, C.L., C.C. King and D.Y. Chao et al., 2005. Laboratory diagnosis of dengue virus infection, current and future perspectives in clinical diagnosis and public health. J. Microbiol. Immunol. Infect., 38: 5-16.
43. Chutinimitkul. S., S. Payungporn and A. Theamboonlers et al., 2005. Dengue typing assay based on real-time PCR using SYBR Green I. J. Virol. Methods, 129: 8-15.

44. Drosten, C., M. Panning and S. Guenther et al., 2002. False-negative results of PCR assay with plasma of patients with severe viral hemorrhagic fever. J. Clin. Microbiol., 40: 4394-4395.

45. Gunther, S., M. Asper and C. Roser et al., 2004. Application of real-time PCR for testing antiviral compounds against Lassa virus, SARS coronavirus and Ebola virus in vitro. Antiviral Res., 63: 209-215.

46. Aitichou, M., S.S. Saleh, A.K. McElroy et al., 2004. Identification of Dobrava, Hantaan, Seoul, and Puumala viruses by one-step real-time RTPCR. J. Virol. Methods, 124: 21-26.

47. Schalk, J.A., C. van den Elzen and H. Ovelgonne et al., 2004. Estimation of the number of infectious measles viruses in live virus vaccines using quantitative real-time PCR. J. Virol. Methods, 117: 179-187.

48. Pang. X.L., B. Lee and N. Boroumand et al., 2004. Increased detection of rotavirus using a real-time reverse transcription-polymerase chain reaction (RT-PCR) assay in stool specimens from children with diarrhea. J. Med. Virol., 72: 496-501.

49. Schwarz, B.A., R. Bange and T.W. Vahlenkamp et al., 2002. Detection and quantitation of group A rotaviruses by competitive and real-time reverse transcription-polymerase chain reaction. J. Virol. Methods, 105: 277-285.

50. Kang, G., M. Iturriza-Gomara and J.G. Wheeler et al., 2004. Quantitation of group A rotavirus by real-time reverse-transcription-polymerase chain reaction, correlation with clinical severity in children in South India. J. Med. Virol., 73: 118122.

51. Becquart, P., V. Foulongne and J. Willumsen et al., 2006. Quantitation of HIV-1 RNA in breast milk by real-time PCR. J. Virol. Methods, 133: 109-111.

52. Gueudin, M. and F. Simon, 2005. Plasma RNA viral load in HIV-1 group $\mathrm{O}$ infection by realtime PCR. Methods Mol. Biol., 304: 221-228. 